\title{
STOPPING AND STRAGGLING OF SLOW ATOMS IN ELECTRON GAS
}

\author{
M. MONETA* \\ University of Eódź, Chair of Solid State Physics \\ Pomorska 149, 90-236 Łódź, Poland
}

(Received July 7, 1997; revised version September 23, 1997)

\begin{abstract}
New analytical formulas for the electronic stopping power and the energy loss straggling of low velocity heavy atoms in the degenerate electron gas are calculated within the dielectric function method. The stopping and straggling effective charges of a projectile were analyzed. They are found to differ each other and to depend on the electron gas density $r_{s}$, on the projectile atomic number $Z_{i}$ and on the projectile degree of ionization $\zeta$.
\end{abstract}

PACS numbers: $71.45 . \mathrm{Gm}$

\section{Introduction}

Since the probability for capture of electrons is high at low ion's velocity, slow ions move or stop in matter almost completely neutralized. The knowledge of the stopping power and energy loss straggling for this atoms are important in analysis of distribution and lattice localization of implanted atoms or in analysis of surface structure. The most important work in this field was done by Lindhard, Winter and Sharff [1-3], Firsov [4] and Hvelplund [5]. A common feature of these theories is the proportionality of the stopping power to the projectile velocity $v$ and the energy loss straggling to $v^{2}$. The target and projectile dependences contained in the proportionality factor are theory dependent and they are different. As far as the author knows, a detailed derivation of the basic Lindhard-Sharff formula has not been presented. Also $r_{s}$ dependence of the straggling has not been determined analytically. In the present calculations stopping and straggling of low velocity projctiles are treated in a unified manner, and in both cases analytical formulas are found. In this paper $m_{\mathrm{e}}, e, a_{0}, v_{0}, \hbar$ are the electron rest mass, the elementary charge, the Bohr radius, the Bohr velocity and the Planck constant divided by $2 \pi$, respectively. Atomic units are used throughout.

\section{Calculation procedure}

The conduction electrons of a solid screen the quasi-static electric potential of an atom due to dielectric response. A volume parameter of bound electron can be determined statistically. Provided the speed of the atom is lower than the Fermi

*E-mail: moneta@krysia.uni.lodz.pl. 
velocity $v_{\mathrm{F}}$, this screening can be approximately described in terms of the screened Coulomb potentials between electrons $V_{\text {ee }}$, and between electron and nucleus $V_{\text {ne }}$ of the following form [6-9]:

$$
V_{\mathrm{ne}}(r)=-\frac{Z_{i} e^{2}}{r} \exp \left(-k_{\mathrm{TF}} r\right), \quad V_{\mathrm{ee}}(r)=\frac{e^{2}}{r} \exp \left(-k_{\mathrm{TF}} r\right)
$$

where the Thomas-Fermi wave number $k_{\mathrm{TF}}$ is related to the electron gas density $n$, or to the one electron radius $r_{s}$ as $k_{\mathrm{TF}}^{2}=4 k_{\mathrm{F}} /\left(\pi a_{0}\right)$ and $k_{\mathrm{F}}$ is the Fermi wave number $k_{\mathrm{F}}=(9 \pi / 4)^{1 / 3} /\left(a_{0} r_{s}\right)$. The $Z_{i}$ is the projectile atomic number.

When an ion is isolated, the volume parameter $\Lambda$ of the bound electrons cloud on the atom is equal to the Brandt-Kitagawa [6] screening length $\Lambda_{0}=$ $0.56 a_{0} / Z_{i}^{1 / 3}$. In a solid this parameter is modified. In order to determine $\Lambda$ we assume stable static conditions. If $N_{i}$ electrons are bound to the ion $\left(\zeta=N_{i} / Z_{i}\right)$ the density $\rho^{\prime}(r)$ can be described by [6]

$$
\rho^{\prime}(r)=\frac{\zeta Z_{i}}{4 \pi \Lambda^{3}} \frac{\Lambda}{r} \exp (-r / \Lambda)=\zeta Z_{i} \rho(r) .
$$

According to the density functional method when the ion moves in a solid the total energy $E$ can be expressed in terms of local electron density $\rho^{\prime}(r)$ as a sum of the kinetic and the potential energies

$$
\begin{aligned}
E= & \frac{3 e^{2}(3 \pi)^{2 / 3}}{10 a_{0}} \int \mathrm{d}^{3} r\left[\rho^{\prime}(r)\right]^{5 / 3}+\int \mathrm{d}^{3} r V_{\mathrm{ne}}(r) \rho^{\prime}(r) \\
& +\frac{1}{2} \int \mathrm{d}^{3} r \mathrm{~d}^{3} r^{\prime} V_{\mathrm{ee}}\left(r-r^{\prime}\right) \rho^{\prime}(r) \rho^{\prime}\left(r^{\prime}\right) .
\end{aligned}
$$

From Eq. (1) and Eq. (2) we get

$$
E=\frac{Z_{i}^{2} e^{2} \zeta}{a_{0}} \frac{a_{0} k_{\mathrm{TF}}}{\kappa}\left[\frac{0.43 \zeta^{2 / 3} \kappa_{0}}{\kappa}-\frac{1}{(1+\kappa)}+\frac{\zeta}{4(1+\kappa)^{2}}\right],
$$

where $\kappa=\Lambda k_{\mathrm{TF}}, \kappa_{0}=\Lambda_{0} k_{\mathrm{TF}}$. From the condition of minimum for the total energy $E$ written as $\partial E / \partial \kappa=0$, we get approximately

$$
\Lambda \approx \Lambda_{0} \zeta^{2 / 3} /\left[1-\kappa_{0}^{1 / 2} / 3\right] \text {. }
$$

The probability of the energy transfer to a degenerate free electron gas from a projectile is described within the random phase approximation by the dielectric function $\epsilon(k, \omega)$. The electronic stopping cross section $S$ and the straggling parameter $\Omega^{2}$ (per free electron) for atoms of velocity $v$ with the bound electron distribution $\rho^{\prime}(r)$ are given as $[1,2,9]$

where

$$
S=\frac{1}{n} \frac{\mathrm{d} E}{\mathrm{~d} x}=\frac{4 \pi e^{4} Z_{i}^{2}}{m v^{2}} L_{1}, \quad \frac{\Omega^{2}}{n x}=\frac{4 \pi e^{4} Z_{i}^{2}}{m v^{2}} L_{2},
$$

$$
L_{m}=\frac{-2 \hbar^{m-1}}{\pi \omega_{\mathrm{p}}^{2}} \int_{0}^{\infty} \frac{\mathrm{d} k}{k}|1-\zeta \bar{\rho}(k)|^{2} \int_{0}^{\infty} \mathrm{d} \omega \omega^{m} \operatorname{Im}\left[\frac{1}{\epsilon(k, \omega)}-1\right] .
$$

In the equations $n$ and $\omega_{\mathrm{p}}=\left(4 \pi n e^{2} / m_{\mathrm{e}}\right)^{1 / 2}=\sqrt{3 / r_{s}^{3}}$ denote the free electron density and the plasma frequency, respectively. The factor $4 \pi e^{4} /\left(m v^{2}\right)=$ $4 \pi e^{2} a_{0}\left(v_{0} / v\right)^{2}$. The form factor $\bar{\rho}(k)=\int \mathrm{d}^{3} r \rho(r) \exp (-\mathrm{i} k r)=1 /\left[1+(\Lambda k)^{2}\right]$ is the Fourier transform of the spatial electron distribution of Eq. (2). 


\section{Results and discussion}

We carried out calculations for ions moving slowly in the uniform electron gas at rest. Analytical results have been derived for the stopping power and for the energy loss straggling for the gas described by Lindhard's dielectric function $\epsilon(u, z)=1+\left(\chi^{2} / z^{2}\right)\left[f_{1}(u, z)+\mathrm{i} f_{2}(u, z)\right][1,2]$ in terms of the dimensionless variables $z=k /\left(2 k_{\mathrm{F}}\right), u=\omega /\left(k v_{\mathrm{F}}\right)$ and $\chi^{2}=r_{s} /\left[\pi(9 \pi / 4)^{1 / 3}\right]$. For low velocity ions the $L_{1}$ and $L_{2}$ functions of Eq. (7) are expressed as

$$
L_{m}=\left(3 E_{\mathrm{F}}\right)^{m-1}\left(\frac{v}{v_{\mathrm{F}}}\right)^{m+1} \int_{0}^{1} \mathrm{~d} z z^{m+2} \frac{\left|1-\zeta \rho\left(2 k_{\mathrm{F}} A z\right)\right|^{2}}{\left(z^{2}+\chi^{2} f_{1}(0, z)\right)^{2}},
$$

where $E_{\mathrm{F}}=(9 \pi / 4)^{2 / 3} /\left(2 r_{s}^{2}\right)$. For $f_{1}(u, z)$ the following expansion $f_{1}(0, z)=1-$ $z^{2} / 3$ can be used. In this case the denominator in Eq. (8) reads $\left[z^{2}+\chi^{2}(1-\right.$ $\left.\left.z^{2} / 3\right)\right]^{2}=\left(\chi^{2} / \chi^{\prime 2}\right)^{2}\left(z^{2}+\chi^{\prime 2}\right)^{2}$ where $\chi^{\prime 2}=\chi^{2} /\left(1-\chi^{2} / 3\right)$. For real metals $1.5<$ $r_{s}<5.8$ therefore $0.5<\chi<0.98$ and $0.52<\chi^{\prime}<1.19$. In the following, in order to simplify notation, we will use the symbol $\chi^{2}$ instead of $\chi^{\prime 2}$. In the calculations, however, we use $\chi^{\prime 2}$.

(1) For the bare atomic nucleus, when $\zeta=0$, from Eq. (8) we get

$$
\begin{aligned}
& L_{1}=\left(\frac{v}{v_{\mathrm{F}}}\right)^{3}\left[0.5\left(-\frac{1}{1+\chi^{2}}+\ln \left(1+\frac{1}{\chi^{2}}\right)\right)\right], \\
& L_{2}=3 E_{\mathrm{F}}\left(\frac{v}{v_{\mathrm{F}}}\right)^{4}\left[1+0.5\left(\frac{\chi^{2}}{1+\chi^{2}}-3 \chi \arctan \frac{1}{\chi}\right)\right] .
\end{aligned}
$$

(2) For a neutral atom $(\zeta=1)$, when the Fourier transform of the electron distribution Eq. (2) is $\bar{\rho}(y z)=1 /\left[1+(y z)^{2}\right]$, where $y=2 k_{\mathrm{F}} \Lambda$ we get

$$
\begin{aligned}
L_{1} & =\left(\frac{v}{v_{\mathrm{F}}}\right)^{3}\left\{\frac { 0 . 5 } { ( \chi ^ { 2 } y ^ { 2 } - 1 ) ^ { 2 } } \left(\frac{-\chi^{4} y^{4}}{\chi^{2}+1}-\frac{y^{2}}{y^{2}+1}\right.\right. \\
& \left.+\frac{1}{\chi^{2} y^{2}-1}\left[\left(\chi^{2} y^{2}-3\right) \chi^{4} y^{4} \ln \left(1+\frac{1}{\chi^{2}}\right)+\left(3 \chi^{2} y^{2}-1\right) \ln \left(1+y^{2}\right)\right]\right\} \\
L_{2} & =3 E_{\mathrm{F}}\left(\frac{v}{v_{\mathrm{F}}}\right)^{4}\left\{1+\frac{0.5}{\left(\chi^{2} y^{2}-1\right)^{2}}\left(\frac{\chi^{6} y^{4}}{1+\chi^{2}}+\frac{1}{1+y^{2}}\right.\right. \\
& \left.\left.+\frac{1}{\chi^{2} y^{2}-1}\left[\chi^{5} y^{4}\left(7-3 \chi^{2} y^{2}\right) \arctan \frac{1}{\chi}+\left(3-7 \chi^{2} y^{2}\right) \frac{1}{y} \arctan y\right]\right)\right\} .
\end{aligned}
$$

(3) For ions carrying $N_{i}$ electrons $\zeta<1$ and using the Fourier transform of the electron distribution in the form $\bar{\rho}(y z)=\zeta /\left[1+(y z)^{2}\right], y=2 k_{F} \Lambda$, we get

$$
\begin{aligned}
& L_{1}=\left(\frac{v}{v_{\mathrm{F}}}\right)^{3}\left[\frac{0.5}{\left(\chi^{2} y^{2}-1\right)^{2}}\left(a_{1}+\frac{1}{\chi^{2} y^{2}-1}\left(b_{1}+c_{1}\right)\right)\right], \\
& L_{2}=3 E_{\mathrm{F}}\left(\frac{v}{v_{\mathrm{F}}}\right)^{4}\left[1+\frac{0.5}{\left(\chi^{2} y^{2}-1\right)^{2}}\left(a_{2}+\frac{1}{\chi^{2} y^{2}-1}\left(b_{2}+c_{2}\right)\right)\right],
\end{aligned}
$$


where the auxiliary functions are defined as

$$
\begin{aligned}
& a_{1}=-\zeta^{2} y^{2} /\left(y^{2}+1\right)-\left(x^{2} y^{2}+\zeta-1\right)^{2} /\left(1+\chi^{2}\right), \\
& b_{1}=\left[-(\zeta-1)^{2}+\chi^{2} y^{2}\left(3-2 \zeta-\zeta^{2}\right)+\chi^{4} y^{4}\left(\chi^{2} y^{2}-3\right)\right] \ln \left(1+1 / \chi^{2}\right), \\
& c_{1}=\left[\zeta \chi^{2} y^{2}+2 \chi^{2} y^{2}+\zeta-2\right] \zeta \ln \left(1+y^{2}\right), \\
& a_{2}=\chi^{2}\left(\chi^{2} y^{2}+\zeta-1\right)^{2} /\left(\chi^{2}+1\right)+\zeta^{2} /\left(y^{2}+1\right), \\
& b_{2}=\left[3(\zeta-1)^{2}+\chi^{2} y^{2}\left(8 \zeta+\zeta^{2}-9\right)+\chi^{4} y^{4}\left(9-2 \zeta-3 \chi^{2} y^{2}\right)\right] \chi \arctan (1 / \chi), \\
& c_{2}=\left(4-\zeta-4 \chi^{2} y^{2}-3 \zeta \chi^{2} y^{2}\right)(\zeta / y) \arctan y .
\end{aligned}
$$

Everywhere above $\chi^{2}=1 /\left(a_{0} \pi k_{\mathrm{F}}\right)=r_{s} /\left[\pi(9 \pi / 4)^{1 / 3}\right]$ and $y=2 k_{\mathrm{F}} \Lambda$. The formulas of Eqs. (13-14) are directly reduced to the cases of a bare atomic nucleus and to the atom by setting $\zeta=0$ and $\zeta=1$, respectively. $L_{1}$ is dimensionless and $L_{2}$ is expressed in atomic Hartree units. For a further analysis we denote the expressions in square brackets of Eqs. (13-14) as $C_{1}$ and $C_{2}$.

The formulas of Eqs. (13-14) as can be expanded in a power series around $\zeta=1$ to yield the first order in $\zeta$ corrections $\Delta L_{m}$ to $L_{m}(\zeta=1)$ which describe an atom

$$
\begin{aligned}
\Delta L_{1} & =\left(\frac{v}{v_{\mathrm{F}}}\right)^{3} \frac{(\zeta-1)}{\left(\chi^{2} y^{2}-1\right)^{2}} \\
& \times\left\{-\frac{\chi^{2} y^{2}}{1+\chi^{2}}-\frac{y^{2}}{1+y^{2}}+\frac{2 \chi^{2} y^{2}}{\chi^{2} y^{2}-1}\left[\ln \left(1+y^{2}\right)-\ln \left(1+\frac{1}{\chi^{2}}\right)\right]\right\}, \\
\Delta L_{2} & =3 E_{\mathrm{F}}\left(\frac{v}{v_{\mathrm{F}}}\right)^{4} \frac{(\zeta-1)}{2\left(\chi^{2} y^{2}-1\right)^{2}}\left\{\frac{1}{1+y^{2}}+\frac{\chi^{4} y^{2}}{1+\chi^{2}}+\frac{2}{\chi^{2} y^{2}-1}\right. \\
& \left.\times\left[\chi^{2} y^{2}\left(5-\chi^{2} y^{2}\right) \arctan \frac{1}{\chi}+\left(1-5 \chi^{2} y^{2}\right) \frac{1}{y} \arctan y\right]\right\}
\end{aligned}
$$

The functions $L_{1}$ and $L_{2}$ of Eqs. (13-14) depend on $y=2 \Lambda k_{\mathrm{F}}$ quadratically around $y=0$. In the first order of expansion we get $L_{1}$ and $L_{2}$ as in Eqs. (9-10), respectively, but multiplied by $(\zeta-1)^{2}$.

The common feature of the present and other results is that at low projectile velocity the stopping power $S$ is proportional to $v$ and the energy loss straggling $\Omega^{2}$ to $v^{2}$. The differences are theory dependent and are related to the coefficient of proportionality $C\left(Z_{i}, r_{s}, \zeta\right)$ which incorporates both the target parameter $r_{s}$ and the projectile parameters $Z_{i}$ and $\zeta$. They cannot be simply separated even after a power expansion. The important point is that we have to consider a stable in time, frozen charge distribution on the projectile. As was shown previously $[10,11]$ the projectile charge in the Fourier space, which contribute to Eqs. (7-8) is a sum of the screening component $[1-\zeta \bar{\rho}(y z)]^{2}$ and the antiscreening component $\left(\zeta / Z_{i}\right)\left\{1-[\bar{\rho}(y z)]^{2}\right\}$. We deal here with slow, heavy projectiles, so the considering of the screening component only is justified. 

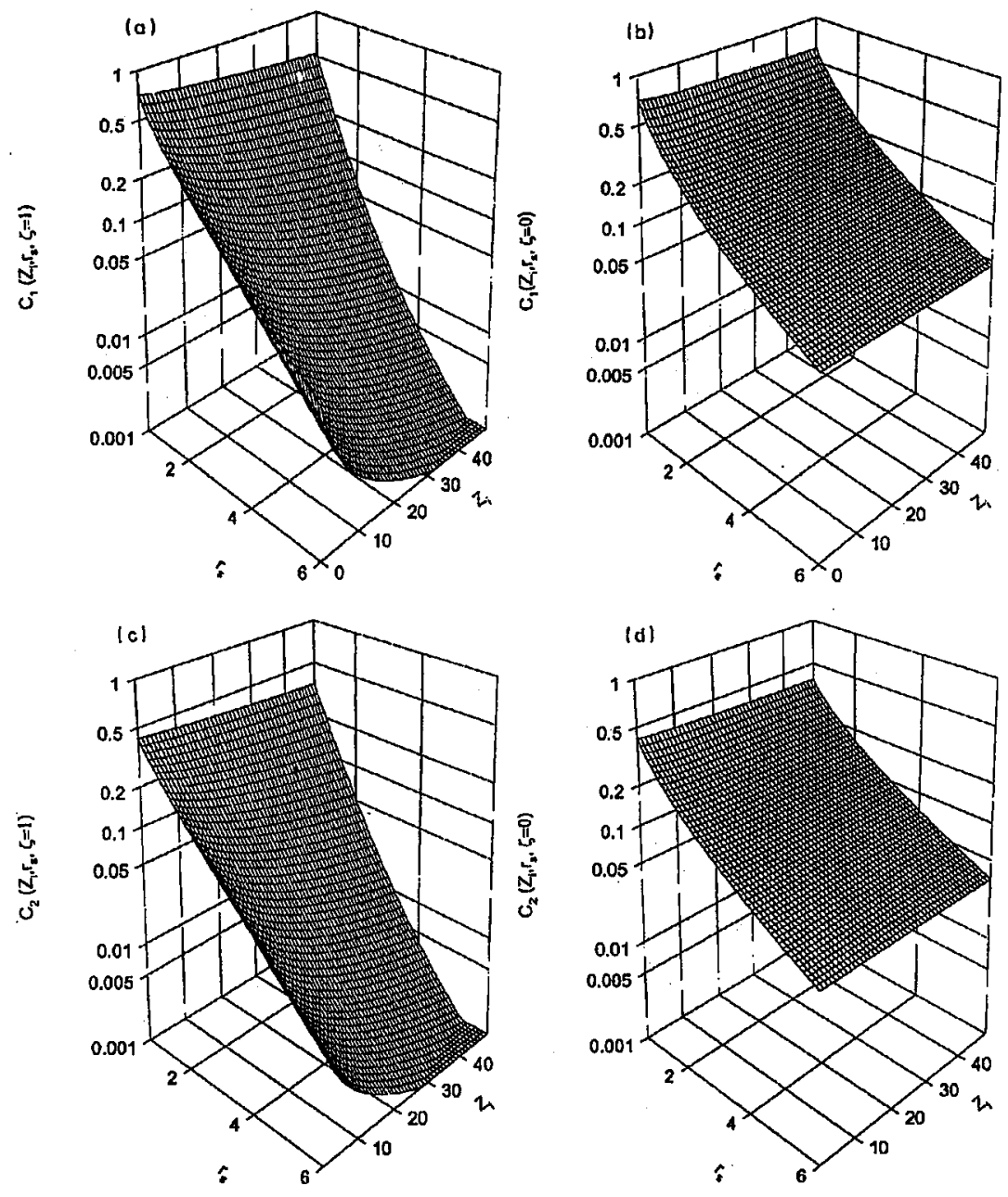

Fig. 1. $\quad r_{s}$ and $Z_{i}$ characteristics of the $C_{1}=L_{1}\left(v_{F} / v\right)^{3}$ function (a) for $\zeta=1$ (atom), (b) for $\zeta=0$ (nucleus) and of the $C_{2}=L_{2}\left(v_{\mathrm{F}} / v\right)^{4} /\left(3 E_{\mathrm{F}}\right)$ function (c) for $\zeta=1$ (atom), (d) for $\zeta=0$ (nucleus).

On analyzing Eq. (5) we find the dependence of the volume parameter $A$ on the electron gas density $r_{s}$. If $r_{s} \rightarrow \infty$ in the vacuum, then $k_{\mathrm{TF}} \rightarrow 0,\left(k_{\mathrm{F}} \rightarrow 0\right)$ and $\Lambda \rightarrow \Lambda_{0}[6]$, as should be. Also $\Lambda$ decreases with a degree of ionization as $\zeta^{2 / 3}$ around $\zeta=1$.

We have drawn results of calculations for $C_{1}$ of Eq. (13) and $C_{2}$ of Eq. (14) in Figs. 1a,b and c,d, respectively, as functions of electron gas density $r_{s}$ and the projectile atomic number $Z_{i}$ for different degrees of ionization $\zeta$. We have found that for dense electron gas $\left(r_{s}=0.5\right)$ the stopping and straggling are almost independent on $\zeta$ and decrease slowly with $Z_{i}$. For a low density $\left(r_{s}=6\right)$ both $C$-functions depend on $\zeta$ very strongly. $C$ 's decrease with $Z_{i}$ for a neutral projectile $(\zeta=1)$, and are almost independent on $Z_{i}$ (compare Eqs. (9-10)) for a bare atomic 

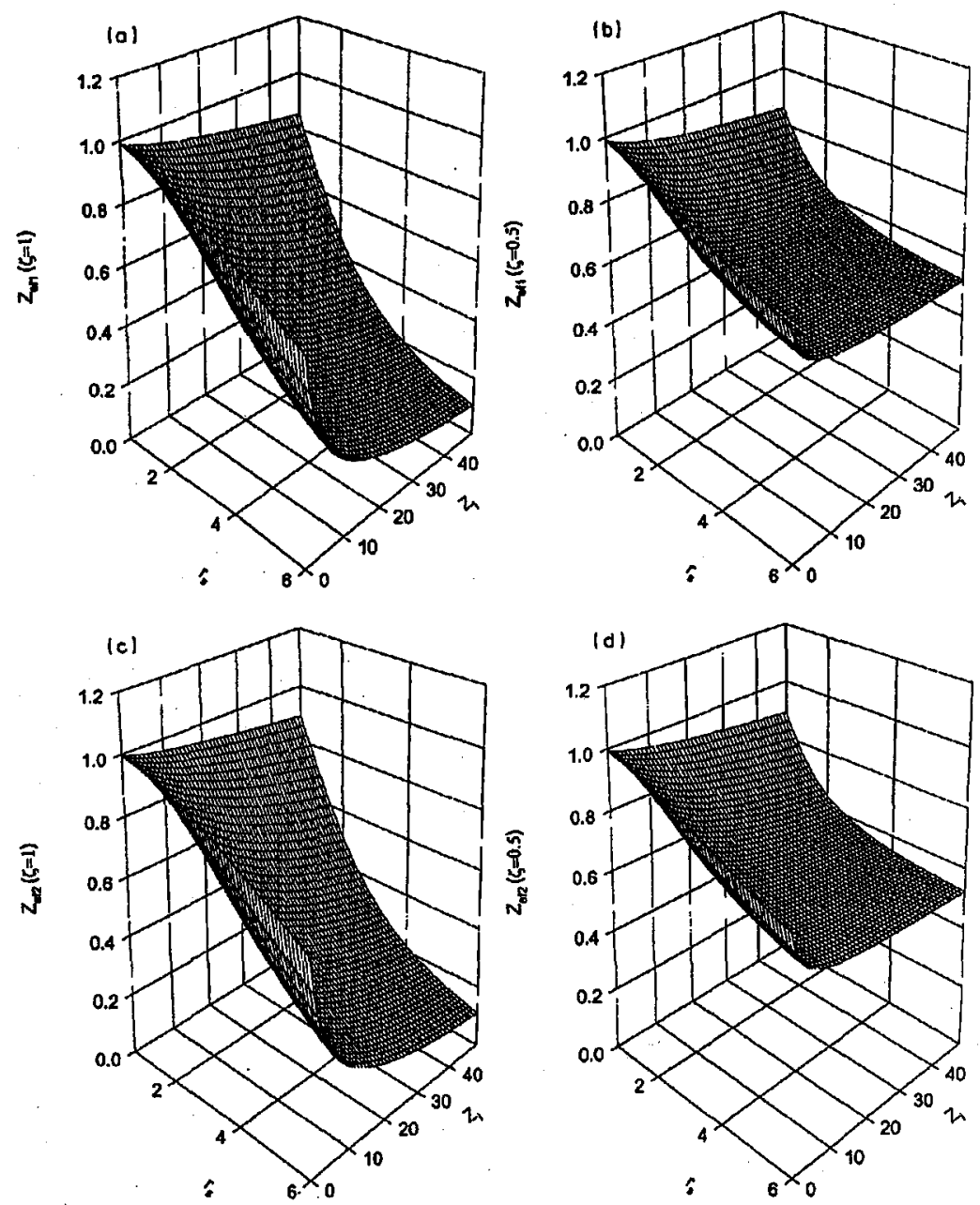

Fig. 2. $r_{s}$ and $Z_{i}$ characteristics of the stopping effective charge $Z_{\mathrm{ef} 1}$ (a) for $\zeta=1$ (atom), (b) for $\zeta=0.5$ and of the straggling effective charge $Z_{\text {ef2 }}$ (c) for $\zeta=1$ (atom), (d) $\zeta=0.5$.

nucleus $(\zeta=0)$. It means that the proportionality of the stopping and straggling to $Z_{i}^{2}$, correct for a point charge, is broken in the case of extended charge, and the projectile excites the medium as a stable charge configuration. The decrease in $C$ 's with $r_{s}$ can be understood by noting that the energy absorbed by the electron gas on collective excitations drops as $r_{s}^{-3 / 2}$ and the number of electrons subjected to the single particles excitations are related to the density of states below the Fermi energy $E_{\mathrm{F}}$.

For the energy loss analysis the concept of effective charge is applied $[10,11]$. It relates stopping and straggling produced by a given projectile to the same characteristics produced by the projectile atomic nucleus. We define the effective charge 
for the stopping $Z_{\text {ef1 }}$ and for straggling $Z_{\text {ef2 }}$ separately as

$$
Z_{\text {efm }}=\sqrt{L_{m}\left(Z_{i}, r_{s}, \zeta\right) / L_{m}\left(Z_{i}, r_{s}, 0\right)}
$$

An independence $Z_{\text {ef }}$ 's on $Z_{i}$ means that the Bethe $Z_{i}^{2}$ scaling is applied for both stopping and straggling. This scaling is related to the same proportion of close and distant collisions in the process of energy transfer to the electron gas. In the static case the result $Z_{\text {ef }}<1$ means that projectile electrons screen the Coulomb potential of the projectile nucleus. However the antiscreening by projectile electrons is neglected [11].

In Fig. 2 we have drawn both effective charges of a neutral $(\zeta=1)$ and half charged $(\zeta=0.5)$ projectiles with different atomic numbers $Z_{i}$ moving slowly in electron gas of density $r_{s}$. Again for dense electron gas the decrease in $Z_{\mathrm{ef}}$ with $Z_{i}$ is slow, and for dilute gas there is a rapid decrease in $Z_{\text {ef }}$ with $Z_{i}$. Also both $Z_{\text {ef }}$ go down with $r_{s}$ much faster for small $Z_{i}$ than for large ones. It means that the stopping power and the energy loss straggling for the extended charge projectile cannot be reduced to the corresponding functions for the proton and some proportionality factor.
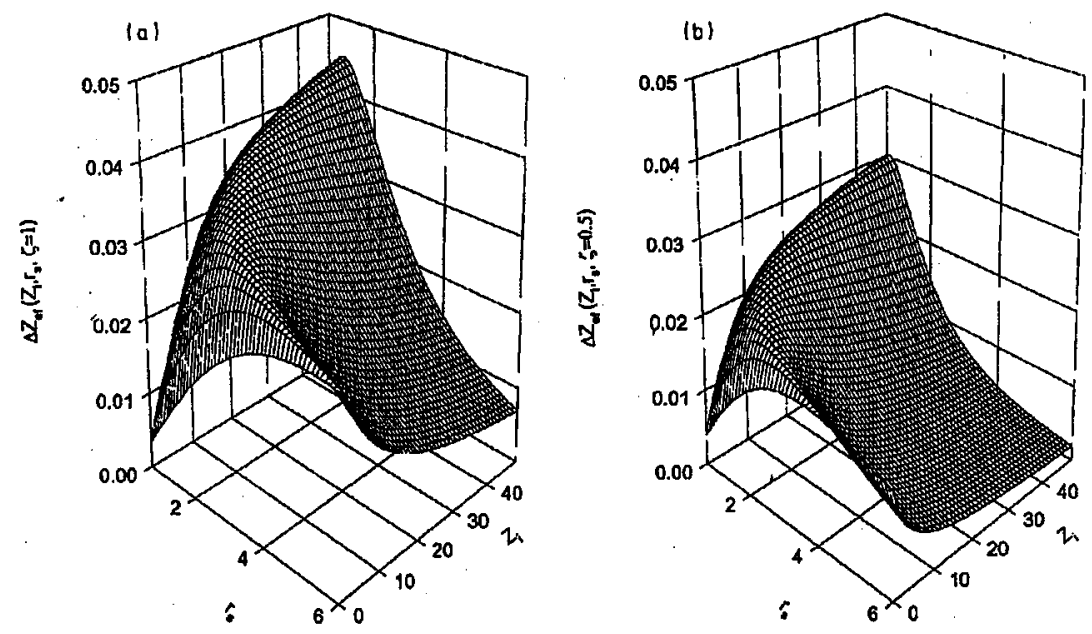

Fig. 3. $r_{s}$ and $Z_{i}$ characteristics of the difference of effective charges $Z_{\mathrm{ef} 2}-Z_{\mathrm{ef} 1}$ for (a) $\zeta=1$ (atom) and (b) $\zeta=0.5$.

An interesting feature is that within the dielectric function method the effective charges extracted from the stopping data and from the straggling data are different. As shown in Fig. 3 the straggling effective charge is larger than the stopping effective charge. The relation $Z_{\mathrm{ef} 2}>Z_{\mathrm{ef} 1}$ holds for all $r_{s}, Z_{i}, \zeta$. Moreover the function $Z_{\text {ef } m}\left(\zeta_{1}\right) / Z_{\text {efm }}\left(\zeta_{2}\right)$ is not constant, but depends on $r_{s}$ and $Z_{i}$. The reason for that is the structure of the integral Eq. (8). It means that there is an additional contribution to the straggling caused by an extended charge projectile. The maximum of the $\Delta Z_{\text {ef }}=Z_{\text {ef } 2}-Z_{\text {ef } 1}$ function forms in the $\left(r_{s}, Z_{i}\right)$ plane a hyperbola. 
For reference we quote the results by Lindhard-Scharff as for the stopping power and the results by Hvelplund as for the energy loss straggling,

$$
\begin{aligned}
& S_{\mathrm{L}}=\mathrm{d} E / N \mathrm{~d} x= \\
& \quad 4 \pi Z_{i}^{2} Z_{\mathrm{t}} 2 Z_{i}^{-5 / 6} /\left(Z_{i}^{2 / 3}+Z_{\mathrm{t}}^{2 / 3}\right)^{3 / 2}\left(v / v_{0}\right) \quad \text { [Hartree } a_{0}^{2} / \text { atom] } \\
& \Omega_{\mathrm{H}}^{2}=\Omega_{\mathrm{B}}^{2} \sqrt{1+13 r_{s} / \pi \alpha}\left(r_{s}^{2} / \alpha^{2}\right)\left(v / v_{0}\right)^{2} \quad \text { [Hartree } a_{0}^{2} / \text { atom], }
\end{aligned}
$$

where $\Omega_{\mathrm{B}}^{2}=4 \pi Z_{i}^{2} Z_{\mathrm{t}} N \mathrm{~d} x$ is the high velocity Bohr straggling, $N$ is the density of target atoms and $Z_{t}$ is the target atomic number. In order to compare these formulas with the present results we should multiply Eqs. (13-14) by a number of free electrons per atom calculated from the plasma frequency $\omega_{\mathrm{p}}$. However, even in this case they remain different. The main differences are related to the fact that the reference formulas were derived for a point charge projectile, and they take into account all the electrons of the target hidden in the combined parameter $Z_{\mathrm{t}}$, whereas the present formulas are derived for extended charge projectile and deal only with the free electron gas. For a point charge moving slowly in the electron gas we get directly Eq. (9) derived by Lindhard [1].

\section{Conclusions}

New analytical formulas for the electronic stopping power and the energy loss straggling of free electron gas for low velocity projectile were derived. The statistical description for the bound electrons was used. The dependence of the effective ion charges on the target electron gas density $r_{s}$ and on the projectile atomic number $Z_{i}$ and the degree of ionization $\zeta$ was discussed. The differences between the stopping and the straggling effective charges were found.

\section{Acknowledgment}

Financial support from University of Łódź, grant 505/576 (1997) is gratefully acknowledged.

\section{References}

[1] J. Lindhard, K. Dam. Vid. Selsk. Mat. Fys. Med 28, No. 8 (1954).

[2] J. Lindhard, A. Winter, K. Dam. Vid. Selsk. Mat. Fys. Med 34, No. 4 (1964).

[3] J. Lindhard, M. Scharf, Phys. Rev A 4, 562 (1971) and K. Dam. Vid. Selsk. Mat. Fys. Med 27, No.15 (1953).

[4] O.B. Firsov, Z. Exp. Theor. Fiz. 36, 1517 (1959).

[5] P. Hvelplund, K. Dam. Vid. Selsk. Mat. Fys. Med 38, No. 4 (1971).

[6] W. Brandt, M. Kitagawa, Phys. Rev. B 25, 5631 (1982).

[7] D. Pines, Elementary Excitations in Solids, W.A. Benjamin, INC New York, Amsterdam 1963.

[8] M. Moneta, Acta Phys. Pol. B 24, 2033 (1993).

[9] M. Moneta, T. Gwizdalla, J. Czerbniak, L. Wojtczak, T. Kaneko, Nucl. Instrum. Methods Phys. Res. B 69, 146 (1992).

[10] M. Moneta, Acta Phys. Pol. A 89, 581 (1996).

[11] M. Moneta, Nucl. Instrum. Methods Phys. Res. B 115, 340 (1996). 